\title{
Criatividade e Saúde Mental: Uma Revisão da Produção Científica na Ultima Década
}

\author{
Karina da Silva Oliveira ${ }^{1}$ \\ Tatiana de Cássia Nakano \\ Solange Muglia Wechsler \\ Curso de Pós-Graduação em Psicologia como Profissão e Ciência da Pontifícia \\ Universidade Católica de Campinas, Campinas, SP, Brasil
}

\section{Resumo}

A relação entre criatividade e saúde mental é foco de debate há mais de cinco séculos, sendo marcada, nos últimos anos pela mudança de paradigma proporcionada pela Psicologia Positiva, cujo foco volta-se à valorização de aspectos sadios e bem desenvolvidos no indivíduo. Buscando investigar melhor o enfoque que tem sido dado à essa relação, o presente estudo analisou a produção científica versada ao tema, publicada no período de 2004 a 2014 nas bases de dados Scientific Electronic Library Online (SciELO), Periódicos Eletrônicos de Psicologia (PePSIC), Medical Literature Analysis and Retrieval System Online (MEDLINE), PsycARTICLES, Academic Search Premier e Academic Search Elite. A partir da análise de 58 artigos, os resultados indicaram um crescimento não linear das produções, equilíbrio entre estudos do tipo teórico e empírico com metodologia qualitativa, publicados pelas áreas de Psicologia e Medicina com um foco predominantemente psicopatológico. Diante dos achados sugere-se o desenvolvimento de estudos sobre a temática, voltados à investigação dos aspectos sadios presentes na relação entre criatividade e saúde mental dentro da tendência atual da Psicologia Positiva.

Palavras-chave: Criatividade, Psicologia Positiva, Psicopatologia, saúde, saúde mental.

\section{Creativity and Mental Health: A Review of Scientific Production in the Last Decade}

\begin{abstract}
The relationship between creativity and mental health is the focus of debate for more than five centuries, being marked in recent years by the paradigm shift provided by Positive Psychology, whose focus back to the recovery of healthy aspects and well developed in the individual. Seeking further investigate the approach that has been given to this relationship, this study analyzed the scientific production versed to the subject, published from 2004 to 2014 in Scientific Electronic Library Online (SciELO) databases, Periódicos Eletrônicos de Psicologia (PePSIC), Medical Literature Analysis and Retrieval System Online (MEDLINE), PsycARTICLES, Academic Search Premier and Academic Search Elite. From the analysis of 58 articles, the results indicated a non-linear growth of production, balance between the theoretical and empirical studies with qualitative methodology, published by the areas of Psychology
\end{abstract}

Endereço para correspondência: Avenida John Boyd Dunlop, s/n ${ }^{\circ}$, Jd. Ipaussurama, Campinas, SP, Brasil 13060-904. Fone: (19) 3343-6892,Fax: (19) 3343-6891. E-mail: karina_oliv@yahoo.com.br Financiamento: Coordenação de Aperfeiçoamento de Pessoal de Nível Superior (CAPES). 
and Medicine, and having a predominantly psychopathological focus. Given the findings suggest the development of studies on the subject, engaged in the study of the healthy aspects in the relationship between creativity and mental health within the current trend of Positive Psychology.

Keywords: Creativity, Positive Psychology, Psychopathology, health, mental health.

\section{Creatividad y Salud Mental: Una Revisión de la Producción Científica de la Última Década}

\section{Resumen}

La relación entre la creatividad y la salud mental es el centro del debate durante más de cinco siglos, está marcada en los últimos años por el cambio de modelo proporcionado por la Psicología Positiva, cuyo foco de nuevo a la recuperación de los aspectos saludables y bien desarrollado en el individuo. Buscando investigar más a fondo el enfoque que se ha dado a esta relación, este estudio analiza la producción científica versado con el tema, publicado desde 2004 hasta 2014 en las bases de datos Scientific Electronic Library Online (SciELO), Periódicos Eletrônicos de Psicologia (PePSIC), Medical Literature Analysis and Retrieval System Online (MEDLINE), PsycARTICLES, Academic Search Premier y Academic Search Elite. A partir del análisis de 58 artículos, los resultados indicaron un crecimiento no lineal de la producción, el equilibrio entre los estudios teóricos y empíricos con metodología cualitativa, publicado por las áreas de Psicología y Medicina, y que tiene un enfoque predominantemente psicopatológico. Dados los hallazgos sugieren el desarrollo de estudios sobre el tema, que participan en el estudio de los aspectos saludables en la relación entre la creatividad y la salud mental dentro de la tendencia actual de la Psicología Positiva.

Palabras clave: Creatividad, Psicología Positiva, Psicopatología, salud, salud mental.

Historicamente, o conceito de criatividade tem se mostrado bastante complexo, assim como o de saúde mental (Silvia \& Kimbrel, 2010). Consequentemente, a relação estabelecida entre estes aspectos é igualmente ampla e muitas vezes controversa, visto que ora a mesma é considerada positiva, ora é entendida como pejorativa (Fox, 2012). Notadamente em relação à segunda visão, pode-se destacar o fato de que, durante muito tempo, a criatividade mostrou-se associada à algumas características psicopatológicas (Inzelberg, 2013), dentro da afirmação aristotélica na qual defendia-se que a genialidade pressupunha a presença de loucura (Fink, Slamar-Habedl, Unterrainer, \& Weiss, 2012) ou de condições de saúde mental fragilizada, podendo-se citar, como exemplos, diversos profissionais considerados expoentes criativos, tais como Leonardo da Vinci, Ernest Hemingway, Pablo Picasso e Frida Kahlo (Richards, Kinney, Lunde, Benet, \& Merzel, 1988), também considerados excêntricos e com algum diagnóstico psiquiátrico.
Uma breve revisão sobre a criatividade mostra que importantes mudanças na sua definição foram ocorrendo ao longo do tempo. Inicialmente compreendida como um dom divino, cuja inspiração era conferida a um número reduzido de pessoas, de modo que o indivíduo não exercia controle sobre esta característica (Douglas, 1977; Sternberg \& Lubart, 1999), passou a ser concebida, em um outro extremo, como manifestação da loucura no século XVI, de maneira que os indivíduos apresentassem características que destoavam do conceito de normalidade da época, eram isolados da sociedade e considerados ameaçadores (Wechsler, 2008). Posteriormente, por volta dos séculos XVII e XVIII, a aproximação entre cultura, arte e ciência favoreceu o avanço conceitual da criatividade (Ximendes, 2010), de forma que, a partir de então, as definições perpassaram os mais diferentes temas culturais, sociais e científicos, notando-se um aumento significativo no número de pesquisas publicadas.

Desde então, tal característica vem sendo reconhecida e valorizada nos mais diferentes 
contextos sociais (Stein \& Harper, 2012), devido à sua importância para o desenvolvimento completo e sadio do indivíduo (Wechsler, 2008). Como consequência, o interesse pelo seu estudo se ampliou no meio científico nas últimas décadas (Nakano, 2005; Nicolas, 1999; Wechsler, 2004; Wechsler \& Nakano, 2002; Zanella \& Titon, 2005). Vista como uma característica a ser estimulada e valorizada, tanto no meio acadêmico quanto no cientifico e social (Nakano \& Wechsler, 2006; Nakano \& Zaia, 2012), a criatividade tem sido visualizada como um recurso para o enfrentamento dos riscos e desafios atuais, como representante do mais alto grau de saúde emocional mas também como forma de superar as dificuldades impostas pelo meio (Oliveira \& Nakano, 2011). Outros autores destacam aspectos benéficos dessa característica, podendo-se citar a possibilidade de alcançar consciência sobre suas potencialidades, a liberdade pessoal e autonomia (Sakamoto, 2000), sua compreensão enquanto um bem social (De La Torre, 2005) e enquanto capacidade de produção de novos conhecimentos (Virgolim, 2007). Dessa maneira, o pensamento criativo tem sido, frequentemente, considerado um diferencial (Lins \& Miyata, 2008), de modo a justificar a importância social e pessoal dessa característica.

Dentre as definições mais aceitas atualmente, encontra-se aquela que define a criatividade como "um processo de sentir deficiências em uma informação, formular hipóteses ou adivinhações sobre estas deficiências, testar e revisar suas hipóteses, e, finalmente, comunicar os resultados encontrados" (Torrance, 1965, p. 8), o qual resulta "numa novidade que é aceita como útil, convincente ou satisfatória por um significativo grupo de pessoas, num determinado período" (Stein, 1994, p. 8), adotada como base para o presente estudo.

O segundo conceito enfocado, saúde mental, é definido como um estado de bem estar que favoreça o desenvolvimento do potencial dos indivíduos, a fim de que estes sejam capazes de realizar o enfrentamento das situações de estresse cotidiano, capazes de trabalhar de modo produtivo e frutífero, estando aptos à contribuírem com suas comunidades (World Health Organiza- tion, http:/www.who.int/features/factfiles/mental_health/en/). De acordo com a Organização Mundial de Saúde (OMS), a saúde mental caracteriza-se como um estado de completo bem- estar físico, mental e social, não se restringindo somente à ausência de doença (http://www.who. int/about/definition/en/print.html).

Apoiado neste referencial, o conceito de saúde mental é compreendido como parte inerente ao desenvolvimento humano, no qual há inter-relações de aspectos físicos, ambientais, sociais, políticos, psicológicos e de demais esferas do cotidiano (Sequeira et al., 2014). Por esta razão, Lluch (2003), ao apresentar uma definição voltada ao tema, afirma que a saúde mental é um estado de bom funcionamento dos indivíduos, no qual há promoção de forças, virtudes e otimização de seu potencial. Assumir a saúde mental como referencial implica em uma postura profissional e teórica que não se restringe à perspectiva de cura e patologia (Heloani \& Capitão, 2003), mas sim, uma postura preocupada com o estudo das qualidades individuais, sociais, emocionais, dentre outras, que auxiliem na proteção e no desenvolvimento dos potenciais humanos (Sequeira et al., 2014). Nesse sentido, Ribeiro (2015) ainda afirma que o conceito, tal qual apresentado, tem como fundamento a crença de que a saúde seja um princípio essencial para o desenvolvimento da felicidade, das relações humanas harmoniosas e seguras.

A questão que se apresenta ao se enfocar a relação entre criatividade e saúde mental ampara-se, segundo Giliam (2013), no argumento de que seja improdutivo apoiar-se em exemplos isolados, tais como personalidades reconhecidamente criativas que apresentavam problemas psicopatológicos, para perpetuar o estereótipo do cientista ou do artista louco, uma vez que, segundo o autor, tal posicionamento apenas confere um caráter glamoroso, ou mesmo caricato à questão, e não a toca de forma objetiva. Do mesmo modo, Acar e Runco (2012) argumentam que esse é um debate bastante delicado visto que ambos os conceitos são profundamente complexos e em muitos aspectos podem influenciar-se mutuamente, já que é possível que a criatividade influencie a saúde mental e vice-versa. Assim, 
Kaufman (2005) afirma que, do ponto de vista da investigação cientifica, é possível encontrar diversos estudos e grandes debates que sugerem a relação entre criatividade e saúde mental, ainda que não se faça presente um consenso quanto à influência dos aspectos envolvidos. Em um dos extremos, Fink et al. (2012) sugerem que a loucura é o preço a ser pago pelo talento humano mais sublime, colocando a questão da relação entre criatividade e saúde mental de forma bastante definitiva.

Diferentes estudos com esse objetivo podem ser citados, conduzidos em diferentes populações e diferentes quadros diagnósticos. Em adultos, o foco volta-se, principalmente, aos transtornos de personalidade e de humor (Acar \& Runco, 2012; Silvia \& Kimbrel, 2010), podendo-se citar exemplos de pesquisas que investigaram a criatividade em pacientes com transtorno bipolar (Richards et al., 1988), ansiedade (Feldhusen, Denny, \& Condon, 1965), dependência de drogas (Fink et al., 2012), psicoticismo (Acar \& Runco, 2012), hipomania (Furnham, Batey, Anand, \& Manfield, 2008), ansiedade e depressão (Silvia \& Kimbrel, 2010) e transtorno bipolar (Simeonova, Chang, Strong, \& Ketter, 2005). Em crianças e adolescentes, os quadros mais comumente enfocados referem-se ao autismo (Pereira, 2006), Transtorno de Déficit de Atenção e Hiperatividade (Csikszentmihalyi, 1996; Germani, 2006; Ourofino \& Fleith, 2005), problemas de aprendizagem (Dias \& Enumo, 2006; Dias, Enumo, \& Azevedo, 2004; Sousa, 2009) e especificamente, dislexia (Alves \& Nakano, 2014; Chakravarty, 2009; Çorlu, Özcan, \& Korkmazlar, 2009; Tafti, Hameedy \& Baghal, 2009).

Sob a perspectiva desses estudos, Krentzman (2013) afirma que na última década, as pesquisas em psicologia assumiram duas vertentes distintas, mas não desassociadas, sendo uma caracterizada pelo interesse na doença e na psicopatologia, e a outra voltada aos aspectos que garantem o desenvolvimento saudável do indivíduo, chamada de Psicologia Positiva. Para Carr (2012), a Psicologia Positiva caracteriza-se por ser uma área de estudos avançados que provocou uma série de mudanças relevantes para a prática em Psicologia, dado o fato de abandonar o olhar voltado apenas ao tratamento da patologia, para enfocar também o desenvolvimento saudável de indivíduos e comunidades. Dentre as habilidades a serem valorizadas, a criatividade pode ser incluída.

A criatividade, enquanto uma característica psicológica, também foi afetada por esse olhar, passando, ao longo da última década, a ser foco de estudos que a colocaram como uma característica positiva e relevante para o desenvolvimento humano (Charyton, Hutchinson, Snow, Rahman, \& Elliot, 2009). Dessa forma, a maior parte dos estudos têm identificado a criatividade como uma característica presente em todos os indivíduos, alterando-se somente seu nível, ou ainda, a intensidade de sua expressão (Beghetto \& Kaufman, 2007; Kaufman \& Beghetto, 2009; Kaufman, Beghetto, \& Pourjalali, 2011), de modo a ampliar o reconhecimento e análise das diversas formas de expressão criativa (Nakano \& Wechsler, 2012). Desse modo, a influência da criatividade nas mais diversas esferas (social, familiar, educacional, trabalho, dentre outras) vem sendo, cada vez mais, intensamente investigada. Um grande interesse pelo construto se tem feito notar, nas últimas décadas, no meio científico nacional (Nakano, 2005; Nakano \& Wechsler, 2007; Zanella \& Titon, 2005), sendo importante destacar que, no entanto, a relação entre criatividade e saúde mental tem se mostrado ainda pouco explorada nos estudos brasileiros.

De modo semelhante, a compreensão sobre a díade saúde/doença tem sido revista, dentro de uma preocupação dos profissionais em não apenas tratar a patologia, mas além disso, promover a saúde e o bem estar dos indivíduos (Jacques, 2003), de maneira que se pode afirmar que o conceito de saúde mental também tem sido revisto, dentro da mesma tendência que tem guiado as propostas do modelo da Psicologia Positiva. Diversos movimentos envolvendo a transformação das práticas psiquiátricas, a revisão do modelo organicista de doença mental, humanização dos hospitais psiquiátricos, assim como o desenvolvimento de trabalhos terapêuticos nesses contextos (Lima, 2015) podem ser citados como ilustrativos das mudanças provocadas. 
Diante do exposto, pode-se verificar que a relação entre a criatividade e a saúde mental, torna-se um tema bastante desafiador de estudo, não somente pela complexidade dos temas, mas também pela alteração de paradigma proposto pela Psicologia Positiva. Tendo em vista que o paradigma científico citado tem-se fortalecido na última década, destacou-se este período para realizar a análise da produção científica sobre a relação apresentada. Desse modo, o presente estudo teve como objetivo analisar a produção científica, nacional e internacional, sobre a relação entre criatividade e saúde mental, buscando-se identificar o ano de publicação dos trabalhos, o tipo de estudo, a área de conhecimento e o foco dado à relação.

\section{Método}

O levantamento dos artigos sobre a temática da relação entre criatividade e saúde mental foi realizado nas seguintes bases de dados: SciELO (Scientific Eletronic Library Online), PePSIC (Periódicos Eletrônicos em Psicologia), Academic Search Premier, Academic Search Elite, Medical Literature Analysis and Retrieval System Online (MEDLINE) Complete e PsycARTICLES, as últimas acessadas por meio da EBSCOHost (http://search.ebscohost.com/).

Para a identificação dos estudos, usou-se a combinação das palavras-chave "creativity and mental health" em todas as bases. O período de publicação foi limitado à última década, selecionando-se aqueles entre 2004 a 2014, visto que é nesse período que os estudos científicos têm apresentado a alteração do paradigma da patologia, para adotarem o da potencialidade humana, conforme apontado por Krentzman (2013).

Os resultados obtidos indicaram a presença inicial de 1.217 artigos produzidos, distribuídos nas bases da seguinte forma: 22 na SciELO, 3 na PePSIC, 589 na Academic Search Premier, 586 na Academic Search Elite, 128 na MEDLINE Complete e 24 na PsycARTICLES. Posteriormente, a análise dos resumos destes estudos foi realizada, visando-se identificar a existência da associação dos conceitos de criatividade e saúde mental. Por meio deste critério, foram excluídos os trabalhos que enfocavam apenas um dos conceitos, sendo que, dos 1.217 trabalhos encontrados inicialmente, 58 foram selecionados, sendo quatro provenientes do SciELO, dois da PePSIC, oito da PsycARTICLES e 44 da MEDLINE Complete. Os trabalhos foram analisados quanto ao ano da publicação, ao âmbito nacional ou internacional, ao tipo de estudo (empírico ou teórico), área de aplicação, público alvo, temática estudada e ao paradigma envolvido.

\section{Resultados e Discussão}

A fim de verificar como o interesse acerca da temática tem se manifestado ao longo do período de tempo selecionado para esse estudo, realizou-se a análise da frequência e da distribuição dos artigos por ano de publicação, considerando o total de artigos $(n=58)$, cujos dados podem ser visualizados na Figura 1.

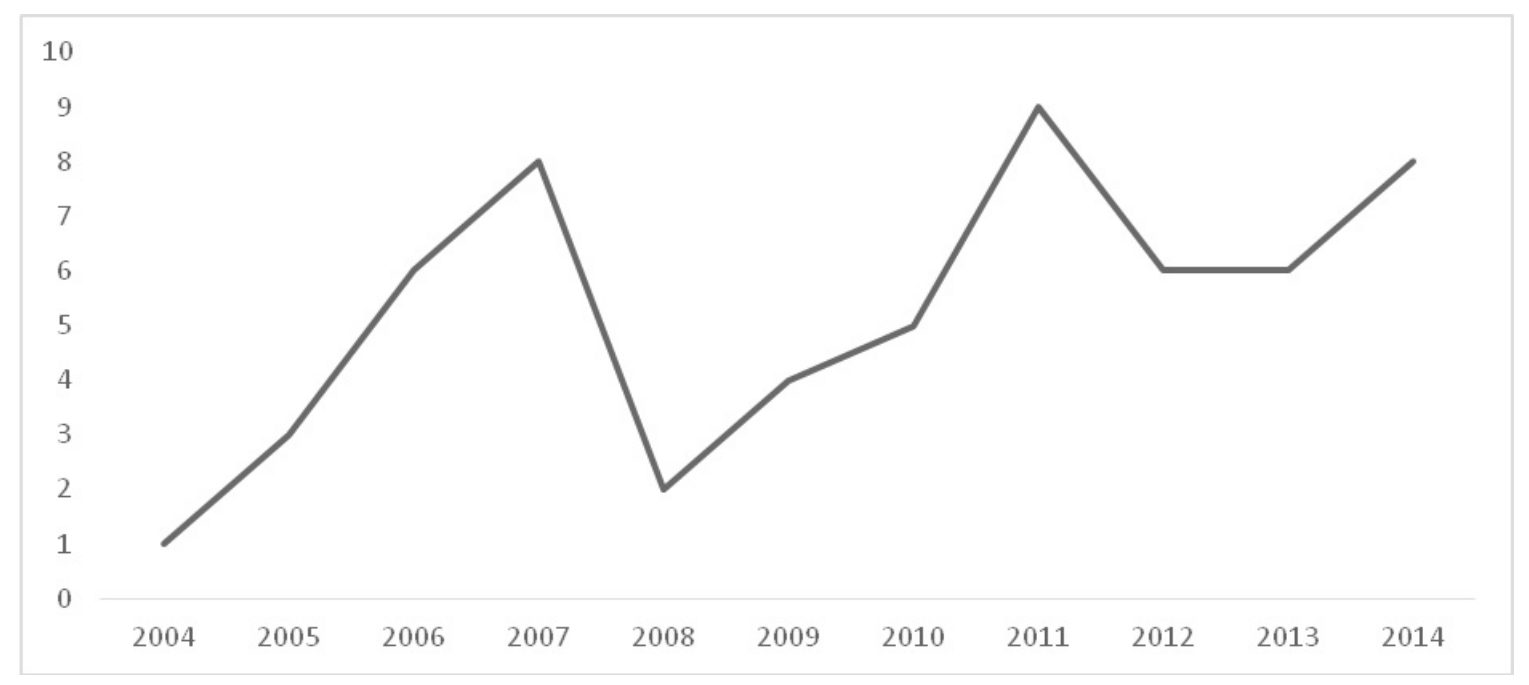

Figura 1. Frequência de artigos publicados, por ano, no período de 2004 a 2014. 
Os dados apontam para um crescimento não linear das produções, sendo possível identificar picos de produção nos anos de $2007(n=8)$ e de 2011 ( $n=9$ ). Em contrapartida, os anos de 2004 $(n=1)$ e $2008(n=2)$, apresentam a menor frequência de artigos sobre o tema. Nota-se ainda uma pequena atenção à temática, visto que 58 trabalhos foram encontrados dentro do período de uma década, o que representa, em média, pouco mais de cinco artigos por ano. Tal procedimento demonstrou que a relação entre criatividade e saúde mental, enquanto conceitos associados, caracteriza-se como um tema de interesse em expansão, tal qual sugerido por Krentzman (2013), ainda que essa afirmação esteja voltada à realidade e interesses da comunidade científica internacional. O que se vê é que tal situação repete-se na análise das publicações nacionais, visto que, conforme observa-se na Figura 1, um crescimento vem sendo notado a partir de 2008 .

Posteriormente os trabalhos foram analisados sob a perspectiva de publicações internacionais $(89,65 \%)$ e nacionais $(10,35 \%)$. Considerando-se esse dado e o ano de publicação dos artigos, ficam evidentes as diferenças quanto ao interesse no tema. Verificou-se que $89,65 \%$ dos trabalhos encontrados compõem as publicações internacionais, sendo que foram encontrados artigos em todos os anos da década investigada. As publicações nacionais perfazem $10,35 \%$ dos estudos analisados, tendo-se encontrado artigos nos anos de $2006(n=2), 2007(n=1), 2010(n=1)$ e $2011(n=2)$.

O segundo critério utilizado para a análise dos resultados, deu-se pelo tipo de estudo utilizado nos artigos, os quais foram agrupados em três categorias: empírico com metodologia qualitativa $(n=22)$, empírico com metodologia quantitativa ( $n=12)$ e estudo teórico $(n=24)$. Percebe-se que há razoável equilíbrio entre a frequência de publicação de estudos qualitativos (37,9\%) e teóricos $(41,3 \%)$, ainda que se faça notar predominância de estudos do segundo tipo quando considerados todos os trabalhos analisados. Tomando-se a origem dos estudos, as bases nacionais (SciELO e PePSIC) apresentaram predominância de estudos de natureza teórica $(71,4 \%)$ enquanto que, nas bases internacionais, faz-se notar mais estudos do tipo qualitativo $(43,1 \%)$, ainda que uma distribuição mais equilibrada possa ser notada entre os três tipos de metodologias. Tais resultados convergem com os dados que têm sido relatados quando se analisam estudos do tipo estado da arte na temática da criatividade, quando tal característica é tomada isoladamente (Nakano \& Wechsler, 2007; Wechsler \& Nakano, 2002, 2003). Entretanto, nessa mesma área diferentes resultados são obtidos (Oliveira \& Nakano, 2011; Silva \& Nakano, 2012; Wechsler, 1999; Zanella \& Titon, 2005), dependendo do foco e tipo de pesquisa.

Posteriormente, os artigos empíricos, sejam eles qualitativos ou quantitativos, foram analisados em relação ao público alvo da pesquisa e estão apresentados na Tabela 1. Os estudos de enfoque qualitativo representaram, em sua maioria, estudos envolvendo adultos de ambos os sexos $(n=18)$, adultos do sexo masculino $(n=1)$, adultos do sexo feminino $(n=1)$ e estudos com idosos de ambos os sexos $(n=2)$. Por sua vez, nos artigos empíricos com metodologia quantitativa $(n=12)$, foram encontrados estudos realizados com crianças $(n=1)$, adolescentes $(n=2)$, adultos $(n=8)$ e idosos $(n=1)$. Nota-se, nas duas metodologias, a predominância de estudos envolvendo amostra composta por indivíduos adultos e, em menor representação, crianças e idosos. Tais dados confirmam os achados relatados por Nakano e Wechsler (2007) e Zanella e Titon (2005), as quais, em pesquisas de revisão de estado da arte, encontraram que as publicações periódicas voltadas à criatividade são mais frequentemente voltadas à investigação de adultos e crianças. Este dado é parcialmente confirmado pelo presente estudo, já que vinte e seis dos artigos analisados têm adultos como público alvo. A diferença se dá do ponto de vista do enfoque infantil, pois foi encontrado apenas um artigo com esta população. Isto pode estar relacionado com o fato da baixa prevalência de transtornos psiquiátricos na infância, estimados em $9 \%$ a $16 \%$ em países desenvolvidos e $15 \%$ a $21 \%$ em países latinos, segundo apontamentos de Feitosa, Ricou, Rego e Nunes (2011). Os mesmos autores, ainda apontam que outro fator a ser considerado, refere-se à dificuldade na identificação e diagnóstico nessa população. 
Tabela 1

Tipo de Estudo e Público Alvo

\begin{tabular}{|c|c|c|c|c|c|c|c|}
\hline Tipo & Amostra & MEDLINE & PsycARTICLES & SciELO & PePSIC & Total & $\%$ \\
\hline \multirow[t]{4}{*}{ Quantitativo } & Criança & 1 & - & - & - & 1 & 2 \\
\hline & Adolescente & 2 & - & - & - & 2 & 3,5 \\
\hline & Adulto & 7 & - & 1 & - & 8 & 14 \\
\hline & Idoso & 1 & - & - & - & 1 & 2 \\
\hline Total Quantitativo & & & & & & 12 & 20,68 \\
\hline \multirow[t]{4}{*}{ Qualitativo } & Criança & - & - & - & - & 0 & 0 \\
\hline & Adolescente & - & - & - & - & 0 & 0 \\
\hline & Adulto & 17 & 2 & 1 & - & 20 & 34 \\
\hline & Idoso & 2 & - & - & - & 2 & 3,5 \\
\hline Total Qualitativo & & & & & & 22 & 37,93 \\
\hline Teórico & - & 14 & 6 & 2 & 3 & 24 & 41 \\
\hline Total Téorico & & & & & & 24 & 41,37 \\
\hline Total de Estudos & & 44 & 8 & 4 & 3 & 58 & 100 \\
\hline
\end{tabular}

Outra análise realizada teve como objetivo a classificação dos trabalhos de acordo com as áreas do conhecimento de origem, a fim de verificar quais delas têm pesquisado e realizado contribuições para a temática da relação entre criatividade e saúde mental. Tal levantamento indicou um maior número de publicações realizado pela Medicina $(n=25)$ e Psicologia $(n=16)$, sendo importante ressaltar a pluralidade de possibilidades de estudo. Por meio dos temas abordados, pode ser verificado em Medicina, por exemplo, trabalhos voltados à utilização de condutas médicas criativas no atendimento de pacientes com quadros psicopatológicos, assim como estudos de técnicas arte-terapêuticas para a redução de sintomas. Já em Psicologia foram encontrados estudos referentes ao impacto de práticas artísticas visualizadas como estratégia de enfrentamento e de alívio para condições traumáticas. No que diz respeito, às outras áreas que têm apresentado produção sobre a temática, destaca-se o Direito $(n=10)$ com trabalhos voltados à construção de políticas públicas e projetos de lei que alcancem pessoas que possuem quadros psicopatológicos, a fim de favorecer a inserção destas pessoas em programas arte-terapêuticos. A predominância de pesquisas sobre criatividade na área da Psico- logia também foi verificada por Campos, Nakano, Ribeiro e Silva (2014), que, ao analisarem 285 trabalhos sobre criatividade e inovação, ainda que tenham sido encontradas publicações em 15 diferentes áreas de conhecimento. De acordo com as autoras, outras áreas relacionadas às ciências humanas também se fizeram presentes (Educação, Pedagogia, Serviço Social, Artes, Comunicação), bem como às ciências biológicas / saúde (Biologia, Fisioterapia) e ciências exatas (Tecnologia da Informação, Engenharia, Economia, Arquitetura), de modo a se poder afirmar que o interesse pela temática tem se mostrado bastante abrangente. A mesma situação tem sido relatada por outros autores, ao salientarem que a criatividade vem se constituindo em tema de interesse de pesquisadores em diferentes áreas, notadamente Psicologia, Administração, Educação, Engenharia, Filosofia, Economia e Artes (Amabile, 1983; Wechsler, 2008). A ausência da citação explicita à Medicina, encontrada no estudo aqui apresentado, justifica-se diante do fato de que as pesquisas citadas tomaram a criatividade de modo geral ou áreas específicas, como organizacional ou educacional, diferente do foco aqui apresentado, da saúde mental, no qual a Medicina tem se apresentado como importante área. 
Outras áreas de interesse que apresentaram publicações na temática selecionada foram a Enfermagem $(n=3)$, na qual os trabalhos estavam associados à práticas criativas no cuidado junto a pacientes psiquiátricos, Filosofia $(n=1)$ cuja contribuição refere-se ao estudo de características de personalidade e circunstâncias pessoais e ambientais que favorecem o processo de descobertas científicas, Letras $(n=1)$ voltada à análise de romances cujos personagens principais apresentavam quadro de enfermidades psiquiátricas, $\mathrm{Pe}$ dagogia $(n=1)$ cujo estudo era voltado às práticas pedagógicas criativas junto à crianças com experiências traumáticas, e Biologia $(n=1)$ na qual analisou-se a influência de um gene relacionado à expressão de características psicopatológicas e pela manifestação de condutas criativas.
Por fim, os artigos também foram analisados quanto ao tema e enfoque conferido aos estudos, cujos dados são apresentados na Tabela 2. Para isso os trabalhos foram agrupados em duas categorias, segundo a natureza da relação estabelecida entre os conceitos de criatividade e saúde mental: enfoque psicopatológico $(n=35)$ e enfoque de saúde $(n=23)$. Como forma de sistematizar a análise, os critérios apresentados pelo Diagnostic and Statiscal Manual of Mental Disorders 5 - DSM V (http://www.dsm5.org/) foram utilizados para a síntese dos temas abordados, sendo importante destacar que aqueles artigos que não enfocavam diretamente o tratamento de doenças foram classificados a partir do enfoque apresentado pelos autores ao longo dos artigos.

Tabela 2

Tabela Comparativa dos Enfoques

\begin{tabular}{|c|c|c|c|c|c|c|c|}
\hline Paradigma & Temática & MEDLINE & PsycARTICLES & SciELO & PePSIC & Total & $\%$ \\
\hline \multirow{10}{*}{ Psicopatológico } & $\begin{array}{l}\text { Esquizofrenia e dos Transtornos } \\
\text { Psicóticos }\end{array}$ & 9 & 1 & 1 & - & 11 & 19 \\
\hline & Estudo genético & 1 & - & - & - & 1 & 2,5 \\
\hline & Transtornos Bipolares & 2 & - & - & - & 2 & 3 \\
\hline & $\begin{array}{l}\text { Transtornos de Estresses Pós } \\
\text { Traumático }\end{array}$ & 2 & 1 & - & - & 3 & 5 \\
\hline & Transtornos Depressivos & 2 & - & - & - & 2 & 3 \\
\hline & $\begin{array}{l}\text { Transtornos Disruptivos, de auto } \\
\text { controle e de conduta }\end{array}$ & 1 & - & - & - & 1 & 2,5 \\
\hline & Transtornos do Espectro Autista & 1 & - & - & - & 1 & 2,5 \\
\hline & $\begin{array}{l}\text { Transtornos drogatictos e } \\
\text { relacionados a uso de substâncias }\end{array}$ & 2 & - & - & - & 2 & 3 \\
\hline & Transtornos Neurocognitivos & 2 & 2 & 1 & 1 & 6 & 10 \\
\hline & Outros transtornos mentais & 4 & 1 & 1 & - & 6 & 10 \\
\hline \multicolumn{2}{|c|}{ Total Enfoque Psicopatológico } & & & & & 35 & \\
\hline \multirow{5}{*}{ Saúde } & Autonomia & 1 & - & - & - & 1 & 2,5 \\
\hline & Habilidades Sociais & 2 & - & - & - & 2 & 3 \\
\hline & Personalidade Criativa & 5 & 1 & - & - & 6 & 10 \\
\hline & Qualidade de vida & 3 & 1 & - & - & 4 & 7 \\
\hline & Resiliência & 7 & 1 & 1 & 1 & 10 & 17 \\
\hline \multicolumn{2}{|c|}{ Total Enfoque Saúde } & & & & & 23 & \\
\hline \multicolumn{2}{|l|}{ Total Estudos } & 44 & 8 & 3 & 2 & 58 & 100 \\
\hline
\end{tabular}


Os dados mostraram que, de uma forma geral, nos estudos focados na investigação da relação entre os conceitos de criatividade e saúde mental, ainda percebe-se a predominância de uma visão psicopatológica $(60,3 \%)$ superior à porcentagem de estudos com enfoque na visão de saúde $(39,7 \%)$. Tal quadro reflete, na realidade, a situação que perdurou, durante muitos anos, de associação entre criatividade a características psicopatológicas ou sua compreensão de maneira pejorativa, notadamente associada à loucura e doença mental (Fink et al., 2012; Fox, 2012; Inzelberg, 2013). Entretanto, a presença de porcentagem importante de trabalhos com enfoque na saúde mostra que a temática tem acompanhado a mudança de paradigma nas pesquisas, com origem no movimento da Psicologia Positiva, cuja ênfase se faz notar no interesse dos pesquisadores nos aspectos positivos e saudáveis do indivíduo (Carr, 2012), notadamente na última década (Charyton et al., 2009), dada a relevância da criatividade para o desenvolvimento pessoal e profissional (Wechsler, 2008).

$\mathrm{O}$ que se pode notar na Tabela 2 é que, dentre os estudos com enfoque psicopatológico da relação entre criatividade e saúde mental, a maior parte deles podem ser classificados no espectro da esquizofrenia e transtornos psicóticos (18,9\% dos trabalhos). A criatividade de pacientes portadores de outros diferentes diagnósticos também foi foco dos estudos, podendo-se citar, como exemplos, transtornos bipolares, depressão, autismo, estresse, dentre outros. Faz-se notar, nestes trabalhos, um esforço dos pesquisadores no sentido de utilizar os recursos possibilitados pelas condutas criativas para a redução de sintomas psicopatológicos, de maneira que a criatividade e a saúde mental, ainda que de forma dialética, podem ser interpretadas dentro de uma relação de influência mútua (Acar \& Runco, 2012), ainda que uma relação direta de causa e consequência não possa ser determinada (Fox, 2012).

Tais dados concordam com aqueles encontrados na revisão da literatura da temática, a qual tem demonstrado que a associação entre criatividade e transtornos mentais têm sido mais fortemente investigada tomando-se, em adultos, os quadros de psicoticismo, hipomania (Furnham et al., 2008), ansiedade e depressão (Silvia \& Kimbrel, 2010) e transtorno bipolar (Simeonova et al., 2005). Já em crianças e adolescentes, os quadros mais comumente investigados relacionam-se ao autismo (Pereira, 2006), síndrome de Asperger, déficit de atenção e hiperatividade (Cramond, 1994; Ourofino \& Fleith, 2005) e problemas de aprendizagem (Dias \& Enumo, 2006; Dias et al., 2004; Graham \& Sheinker, 1980; Gregg \& Hoy, 1984; Holguin \& Sherrill, 1989; Sousa, 2009; Tarver, Ellsworth, \& Rounds, 1980).

Em relação ao enfoque baseado na visão de saúde, aqueles com foco na resiliência são maioria (17\%), sendo importante também o número de trabalhos voltados à personalidade criativa $(10 \%)$ e qualidade de vida (7\%), ainda que outros aspectos também tenham sido encontrados, tais como autonomia e habilidades sociais. Novamente, os achados corroboram a literatura científica. Especificamente sobre a relação entre criatividade e resiliência, Oliveira e Nakano (2011) apontaram que, embora as duas temáticas venham recebendo atualmente bastante atenção por parte do meio científico, devido principalmente ao fato de serem consideradas mecanismos favoráveis ao desenvolvimento sadio do indivíduo, o que se tem notado é uma carência de estudos nacionais que investiguem essa relação. Tal fato pode ser confirmado na revisão aqui apresentada, na qual somente sete artigos foram encontrados na década investigada. Ainda de acordo com as autoras, o que se pode concluir é que a resiliência tem sido reconhecida como um suporte para a promoção de saúde mental, a qual, aliada à criatividade, poderia ser utilizada como um recurso na busca por respostas mais eficazes e soluções mais adaptadas na presença de um ambiente desfavorável. Tal constatação justifica a necessidade de que essa relação seja melhor investigada a fim de que os conhecimentos dela advindos pudessem ser utilizados na elaboração de programas e projetos de prevenção e intervenção.

O reconhecimento da relação positiva entre criatividade e saúde mental, baseada em um enfoque de saúde, encontra amparo no reconhecimento, mais recente, do conceito de du- 
pla excepcionalidade, indivíduos duplamente excepcionais, mais notadamente pesquisado tomando-se o fenômeno das altas habilidades / superdotação. Esse padrão encontra-se cada vez mais sendo investigado na literatura sob a denominação de "dupla-excepcionalidade", que pode ser mais especificamente definida como a presença de uma alta performance, talento, habilidade ou potencial em alguma área específica, a qual ocorre em conjunto com uma desordem psiquiátrica, educacional, sensorial e/ou física (Pfeiffer, 2013). Envolve a ideia de que o desempenho e a aprendizagem podem caminhar em duas direções, ou seja, pessoas que demonstram capacidades superiores em uma ou mais áreas e que, ao mesmo tempo, apresentariam também deficiências ou diagnósticos psiquiátricos, conforme apontado por Nakano e Siqueira (2012).
Especificamente em relação à criatividade, Alves e Nakano (2015) constataram, após revisão dos estudos sobre dupla excepcionalidade, que, os conhecimentos que podem advir dessa área podem ser utilizados no sentido de promoção e reconhecimento de habilidades que possam estar desenvolvidas em quadros considerados debilitantes, dentro das tendências atuais da Psicologia Positiva.

Buscando-se verificar a existência de relação temporal entre os estudos encontrados, um último levantamento foi realizado, tendo-se como objetivo a classificação dos artigos de acordo com o enfoque (psicopatológico e saúde), por ano de publicação, baseando-se na hipótese de que os últimos seriam mais recentes, de modo a acompanhar a tendência da psicologia positiva. Os resultados podem ser visualizados na Figura 2.

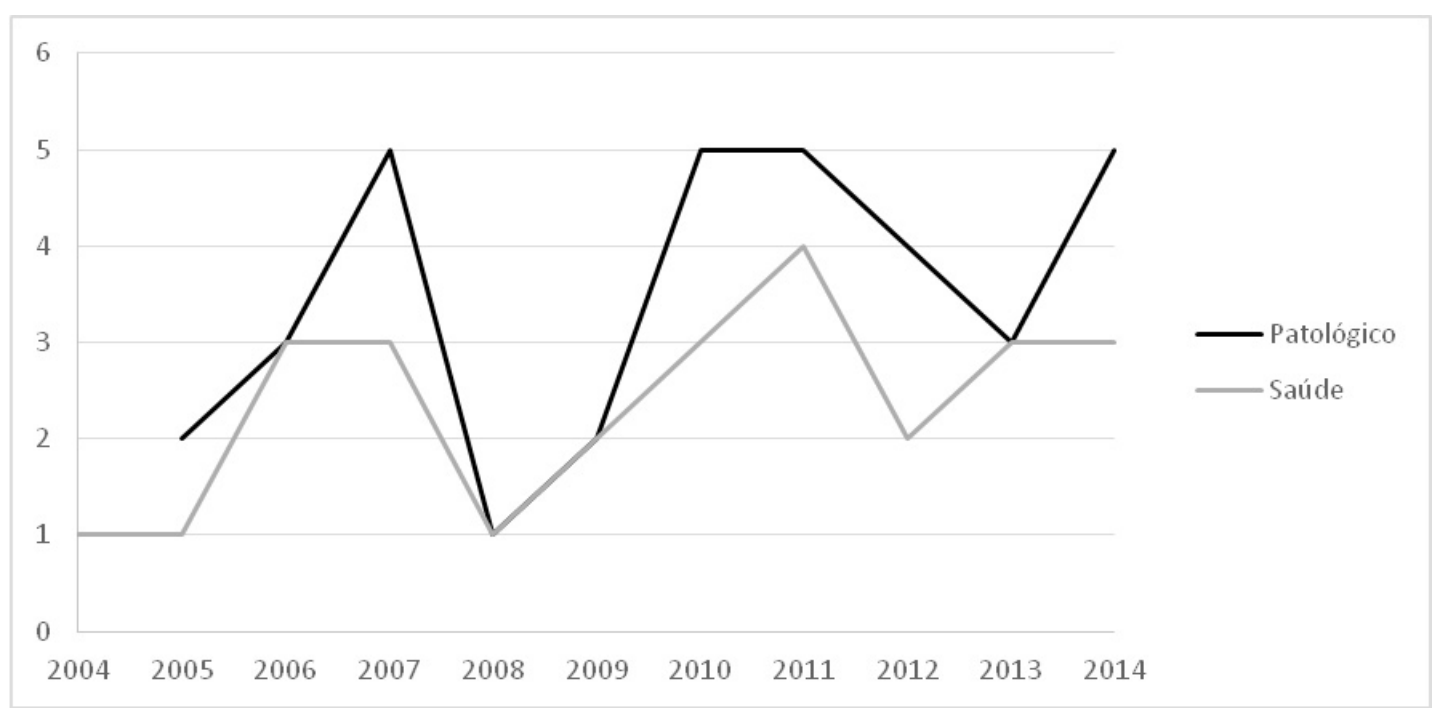

Figura 2. Frequência das publicações a partir da classificação do paradigma apresentado pelo estudo.

Os dados mostraram que, de modo geral, a relação entre criatividade e saúde mental tem sido, tradicionalmente, mais baseada no enfoque patológico. Ainda que um crescimento no enfoque voltado à saúde possa ser notado, principalmente a partir de 2009, o número de pesquisas mostra-se inferior ao primeiro grupo. Um levantamento mais extenso é recomendado a fim de que uma visão mais ampla desse percurso histórico possa ser melhor visualizada, notadamente no período anterior ao surgimento do enfoque da psicologia positiva.

\section{Conclusões}

Se considerarmos que pessoas criativas e pessoas que sofrem de alguns transtornos mentais podem compartilhar características de personalidade e traços cognitivos semelhantes conforme salientado por Fink et al. (2012), a importância do debate da relação entre habilidades criativas e psicopatologias justifica-se. Entretanto, ao realizar o levantamento da produção voltada a compreensão dessa complexa relação, foi possível identificar algumas lacunas que difi- 
cultam o aprofundamento ou mesmo o esclarecimento da questão. Destaque-se, dentre essas, o fato de que ambos conceitos são desafiadores, tanto do ponto de vista das possibilidades de definição, da multiplicidade teórica, da dificuldade em delimitar as populações alvo e do acesso a esses indivíduos, características que fazem desta temática um campo árido e que merece ser melhor investigado, notadamente nas pesquisas nacionais

Não foi pretensão deste estudo concluir qual aspecto é mais determinante na origem e manutenção do outro, seja a criatividade na expressão da patologia ou mesmo a patologia na expressão da criatividade, mas sim verificar se o paradigma da Psicologia Positiva tem, de fato, provocado mudanças na compreensão dessa relação na última década. Os resultados mostraram que a maior parte das produções encontradas ainda mantêm-se associadas à produção do conhecimento voltado ao tratamento da patologia, ainda que o número de estudos voltados à investigação dessa relação dentro de uma visão de saúde venha aumentando, de forma não linear e inconstante, ao longo deste período.

Verifica-se também, que essa relação despertou o interesse de diferentes áreas do conhecimento, as quais têm contribuído para a discussão, assim como favorecido a prática junto aos indivíduos que necessitam do apoio profissional, seja do ponto de vista médico, psicológico e até mesmo na ampliação de direitos. Faz-se notar a possibilidade de um trabalho dentro de uma visão global e multidisciplinar.

No presente estudo, ao optar-se por realizar a análise dos dados a partir de palavras chaves bastante específicas é possível que estudos que tratam da relação de forma indireta não tenham sido contemplados, tais como trabalhos que tratem de características positivas tais como resiliência e adaptação, por exemplo. Do mesmo modo, dado o fato de que muitas bases de dados tornaram-se eletrônicas somente nos últimos anos, muitos trabalhos podem ter sido excluídos da busca. Desta forma, outros estudos devem ser conduzidos a fim de ampliar as compreensões aqui expostas. Sugere-se ainda que outras bases de dados sejam utilizadas em pesquisa futuras, tomando-se não só as publicações periódicas, mas também outras fontes de dados, tais como teses e dissertações, a fim de que os dados aqui relatados possam ser confirmados.

\section{Referências}

Acar, S., \& Runco, M. (2012). Psychoticism and creativity: A meta-analytic review. Psychology of Aesthetics, Creativity, and the Arts, 6(4), 341350. doi:10.1037/a0027497

Alves, R. J. R., \& Nakano, T. C. (2014). Creativity and intelligence in children and without developmental dyslexia. Paideia (Ribeirão Preto), 24(59), 361-369. doi:10.1590/198243272459201410

Alves, R. J. R., \& Nakano, T. C. (2015). A dupla-excepcionalidade: Relação entre altas habilidades/superdotação com a síndrome de Asperger, transtorno de déficit de atenção e hiperatividade e transtornos de aprendizagem. Revista Psicopedagogia, 32(99), 346-360.

Amabile, T. M. (1983). The social psychology of creativity. New York: Springer-Verlag.

Beghetto, R. A., \& Kaufman, J. (2007). Toward a broad conception of creativity: A case for "minic" Creativity. Creativity and the Arts, 1(2), 7379. doi:10.1037/1931-3896.1.2.73

Campos, C. R., Nakano, T. C., Ribeiro, W. J., \& Silva, T. F. (2014). Criatividade e inovação: Uma revisão da produção científica no Brasil. Faculdades do Saber, 1, 168-179.

Carr, A. (2012). Positive Psychology: The science of happiness and human strengths. Psychiatric Rehabilitation Journal, 35(4), 355-358. doi:10.4324/9780203156629

Chakravarty, A. (2009). Artistic talent in dyslexia A hypothesis. Medical Hypotheses, 73(4), 569571. doi:10.1016/j.mehy.2009.05.034

Charyton, C., Hutchison, S., Snow, L., Rahman, M. A., \& Elliot, J. O. (2009). Creativity as an attribute of Positive Psychology: The impact of positive and negative effect on the creative personality. Journal of Creativity in Mental Health, 4(1), 57-66. doi:10.1080/15401380802708791

Çorlu, M., Özcan, O., \& Korkmazlar, Ü. (2009). The meaning of dyslexic's drawings in communication design. Dyslexia, 15(2), 148-154. doi:10.1002/dys.362 
Cramond, B. (1994). The relationship between attention-deficit hyperactivity disorder and creativity? Available from ERIC Document Reproduction Service. (No. ED371495).

Csikszentmihalyi, M. (1996). Creativity: Flow and the psychology of discovery and invention. New York: Harper Collins

De la Torre, S. (2005). Dialogando com a criatividade. São Paulo, SP: Madras.

Dias, T. L., \& Enumo, S. R. F. (2006). Criatividade em crianças com dificuldade de aprendizagem: Avaliação e intervenção através de procedimentos tradicional e assistido. Psicologia: Teoria e Pesquisa, 22(1), 69-78. doi:10.1590/S010237722006000100009

Dias, T. L., Enumo, S. R. F., \& Azevedo, R. R., Jr. (2004). Influências de um programa de criatividade no desempenho cognitivo e acadêmico de alunos com dificuldade de aprendizagem. Psicologia em Estudo, 9, 429-437. doi:10.1590/ S1413-73722004000300011

Douglas, J. H. (1977). The genius of everyman (1): Discovering creativity. Science News, 111(17), $268-270$

Feitosa, H. N., Ricou, M., Rego, S., \& Nunes, R. (2011). A saúde mental de crianças e dos adolescentes: Considerações epidemiológicas, assistenciais e bioéticas. Revista Bioética, 19 (1), $259-275$

Feldhusen, J. F., Denny, T., \& Condon, C. F. (1965). Anxiety, divergent thinking, and achievement. Journal of Educational Psychology, 56(1), 40-45.

Fink, A., Slamar-Halbedl, M., Unterrainer, H. F., \& Weiss, E. M. (2012). Creativity: Genius, madness or a combination of both?. Psychology of Aesthetics, Creativity, and the Arts, 6(1), 11-18. doi:10.1037/a0024874

Fox, N. J. (2012). Creativity and health: An antihumanist reflection. Health, 17(5), 495-511. doi:10.1177/1363459312464074

Furnham, A., Batey, M., Anand, K., \& Manfield, J. (2008). Personality, hypomania, intelligence and creativity. Personality and Individual Differences, 44(5), 1060-1069. doi:10.1016/j. paid.2007.10.035

Germani, L. M. B. (2006). Características de altas habilidades/superdotação e de déficit de atenção/hiperatividade: Uma contribuição à família e à escola (Dissertação de mestrado, Pontifícia
Universidade Católica do Rio Grande do Sul, Porto Alegre, RS, Brasil).

Giliam, T. (2013). Creativity and mental health care. Art \& Science: Arts in Practice, 16(9), 24-30. doi:10.7748/mhp2013.06.16.9.24.e807

Graham, S., \& Sheinker, A. (1980). Creative capabilities of learning-disabled and normal students. Perceptual and Motor Skills, 50(2), 481-482.

Gregg, N., \& Hoy, C. (1984). Learning disabled college students' figural problem solving skills. Available from ERIC Document Reproduction Service. (No. ED289283)

Heloani, J. R., \& Capitão, C. G. (2003). Saúde mental e psicologia do trabalho. São Paulo em Perspectiva, 17(2), 102-108. doi:10.1590/S010288392003000200011

Holguin, O., \& Sherrill, C. (1989). Use of a motor creativity test with young learning disabled boys. Perceptual and Motor Skills, 69(3, Pt 2), 1315-1318

Inzelberg, R. (2013). The awakening of artistic creativity and Parkinson's disease. Behavioral Neuroscience, 127(2), 256-261. doi:10.1037/ a0031052

Jacques, M. G. C. (2003). Abordagens teórico-metodológicas em saúde/doença mental \& trabalho. Psicologia e Sociedade, 15(1), 97-116.

Kaufman, J. C. (2005). The door that leads into madness: Eastern European poets and mental illness. Creativity Research Journal, 17(1), 99103. doi:10.1207/s15326934crj1701 8

Kaufman, J. C., \& Beghetto, R. A. (2009). Beyond Big and Little: The four $\mathrm{C}$ models of creativity. Review of General Psychology, 13(1), 1-12. doi:10.1037/a0013688

Kaufman, J. C., Beghetto, R. A., \& Pourjalali, S. (2011). Criatividade na sala de aula: Uma perspectiva internacional. In S. M. Wechsler \& V. L. T. Souza (Eds.), Criatividade e aprendizagem: Uma perspectiva internacional (pp. 53-72). São Paulo, SP: Loyola.

Krentzman, A. R. (2013). Review of the application of Positive Psychology to substance use, addiction, and recovery research. Psychology of Addictive Behaviors, 27(1), 151-165. doi:10.1037/ a0029897

Lima, M. E. A. (2015). Saúde mental e trabalho. In P. F. Bendassolli \& J. E. Borges-Andrade (Eds.), 
Dicionário de Psicologia do Trabalho e das Organizações (pp. 607-616). São Paulo, SP: Casa do Psicólogo.

Lins, J. S. C., \& Miyata, E. S. (2008). Avaliando a aprendizagem de criatividade em uma oficina pedagógica. Ensaio: Avaliação de Políticas Públicas Educacionais, 16(60), 455-468. doi:10.1590/S0104-40362008000300008

Lluch, M. T. (2003). Construccíon y análisis psicométrico de un cuestionario para evaluar la salud mental positiva. Psicología Conductal, 11(1), 61-78.

Nakano, T. C. (2005). Pesquisa em criatividade: Análise da produção científica do banco de teses da Capes (1996-2001). In G. P. Witter (Ed.), Metaciência e Psicologia (pp. 35-48). Campinas, SP: Alínea.

Nakano, T. C., \& Siqueira, L. G. G. (2012). Validade de conteúdo da Gifted Rating Scale (versão escolar) para a população brasileira. Avaliação Psicológica, 11(1), 123-140.

Nakano, T. C., \& Wechsler, S. M. (2006). Teste Brasileiro de Criatividade Figural: Proposta de instrumento. Interamerican Journal of Psychology, 40,103-110.

Nakano, T. C., \& Wechsler, S. M. (2007). Criatividade: Características da produção científica brasileira. Avaliação Psicológica, 6(2), 261-270.

Nakano, T. C., \& Wechsler, S. M. (2012). Criatividade: Definições, modelos e formas de avaliação. In C. S. Hutz (Ed.), Avanços em avaliação psicológica de crianças e adolescentes II (pp. 327361) São Paulo, SP: Casa do Psicólogo.

Nakano, T. C., \& Zaia, P. (2012). Criatividade e inteligência emocional em crianças: Um estudo relacional. Psico, 43, 388-399.

Nicolas, A. M. N. (1999). Criatividade onde está? Catharsis, 4, 11-12.

Oliveira, M. A., \& Nakano, T. C. (2011). Revisão de pesquisas sobre criatividade e resiliência. Temas em Psicologia, 19, 467-479.

Ourofino, V. T. A. T., \& Fleith, D. S. (2005). Um estudo comparativo sobre a dupla personalidade superdotação/hiperatividade. Avaliação Psicológica, 4(2), 165-182.

Pereira, D. F. (2006). Um estudo sobre o Wartegg como medida de criatividade em seleção de pessoal (Dissertação de mestrado não publicada, Faculdade de Psicologia, Universidade
Federal do Rio Grande do Sul, Porto Alegre, RS, Brasil).

Pfeiffer, S. I. (2013). Serving the gifted: Evidencebased clinical and psycho-educational practice. New York: Routledge.

Ribeiro, J. L. P. (2015). Educação para saúde. Psicologia, Saúde \& Doenças, 16(1), 3-9. doi:10.15309/15psd160102

Richards, R., Kinney, D. K., Lunde, I., Benet, M., \& Merzel, A. P. C. (1988). Creativity in manic-depressives, cyclothymes, their normal relatives, and control subjects. Journal of Abnormal Psychology, 97(3), 281-288. doi:10.1037/0021$-843 X .97 .3 .281$

Sakamoto, C. K. (2000). Criatividade: Uma visão integradora. Psicologia: Teoria e Prática, 2(1), 50-58.

Sequeira, C., Carvalho, J. C., Sampaio, F., Sá, L., Lluch-Canut, T., \& Roldán-Merino, J. (2014). Avaliação das propriedades psicométricas do questionário de saúde mental positiva em estudantes portugueses do ensino superior. Revista Portuguesa de Enfermagem de Saúde Mental, (11), 45-53.

Silva, T. F., \& Nakano, T. C. (2012). Criatividade no contexto educacional: Análise de publicações periódicas e trabalhos de pós-graduação na área da psicologia. Educação e Pesquisa, 38(3), 743759. doi:10.1590/S1517-97022012005000013

Silvia, P. J., \& Kimbrel, N. A. (2010). A dimensional analysis of creativity and mental illness: Do anxiety and depression symptoms predict creative cognition, creative accomplishments, and creative self-concepts?. Psychology of Aesthetics, Creativity, and the Arts, 4(1), 2-10. doi:10.1037/ t05569-000

Simeonova, D. I., Chang, K. D., Strong, C., \& Ketter, T. A. (2005). Creativity in familial bipolar disorder. Journal of Psychiatric Research, 39(6), 623-631. doi:10.1016/j.jpsychires.2005.01.005

Sousa, D. A. (2009). How the gifted brain learns. Thousand Oaks, CA: Corwin Press.

Stein, L. M. (1994). Teste de Desempenho Escolar. São Paulo, SP: Casa do Psicólogo.

Stein, S. M., \& Harper, T. L. (2012). Creativity and innovation: Divergence and convergence in pragmatic dialogical planning. Journal of Planning Education and Research, 32(1), 5-17. doi:10.1177/0739456X11417829 
Sternberg, R. J., \& Lubart, T. I. (1999). The concept of creativity: Prospects and paradigms. In R. J. Sterberg, Handbook of creativity (pp. 3-15). Cambridge, UK: Cambridge University Press.

Tafti, M. A., Hameedy, M. A., \& Baghal, N. M. (2009). Dyslexia, a deficit or a difference: Comparing the creativity and memory skills of dyslexic and nondyslexic students in Iran. Social Behavior and Personality, 37(8), 1009-1016. doi:10.2224/sbp.2009.37.8.1009

Tarver, S. G., Ellsworth, P. S., \& Rounds, D. J. (1980). Figural and verbal creativity in learning disabled and nondisabled children. Learning Disability Quarterly, 3(3), 11-18.

Torrance, E. P. (1965). Rewarding creative behavior: Experiments in classroom creativity. Englewood Cliffs, NJ: Prentice Hall.

Virgolim, A. M. R. (2007). Talento criativo: Expressão em múltiplos contextos. Brasília, DF: Universidade de Brasília.

Wechsler, S. M. (1999). Avaliação da criatividade: Um enfoque multidimensional. In S. M. Wechsler \& R. S. L. Guzzo (Eds.), Avaliação Psicológica: Perspectiva internacional (pp. 231-259). São Paulo, SP: Casa do Psicólogo.

Wechsler, S. M. (2004). Avaliação da criatividade verbal no contexto brasileiro. Avaliação Psicológica, 3, 21-31.
Wechsler, S. M. (2008). Criatividade: Descobrindo e encorajando (3. ed.). Campinas, SP: Laboratório de Avaliação e Medidas Psicológicas, Pontifícia Universidade Católica de Campinas.

Wechsler, S. M., \& Nakano, T. C. (2002). Caminhos para avaliação da criatividade: Perspectiva brasileira. In R. Primi (Ed.), Temas em Avaliação Psicológica (pp. 103-115). Campinas, SP: Instituto Brasileiro de Avaliação Psicológica.

Wechsler, S. M., \& Nakano, T. C. (2003). Produção científica em criatividade: $\mathrm{O}$ estado da arte. Escritos sobre Educação, 2, 43-50.

Ximendes, E. (2010). As bases neurocientificas da criatividade: $O$ contributo da neurociência no estudo do comportamento criativo (Dissertação de mestrado). Recuperado em http://repositorio. ul.pt/handle/10451/7285

Zanella, A. V., \& Titon, A. P. (2005). Análise da produção científica sobre criatividade em programas brasileiros de pós-graduação em psicologia (1994-2001). Psicologia em Estudo, 10, 305316. doi:10.1590/S1413-73722005000200018

Recebido: 20/05/2015

$1^{a}$ revisão: $1^{\circ} 12 / 2015$ Aceite final: 10/12/2015 